\title{
ASYMMETRIC MODES OF THE KELVIN-HELMHOLTZ INSTABILITY IN PROTOSTELLAR JETS
}

\author{
JAMES M. STONE \\ Department of Astronomy, University of Maryland \\ College Park, MD 20742-2421 USA
}

\begin{abstract}
The results of a detailed analysis of the linear properties, nonlinear growth, and saturation of asymmetric modes of the Kelvin-Helmholtz instability in cooling protostellar jet beams are summarized. In the linear regime, cooling can significantly alter the growth rate and wavelength of the most unstable mode in comparison to an adiabatic jet. In the nonlinear regime, sinusoidal oscillations at the maximum growth rate lead to distortions that will be observed as 'wiggles' or 'kinks' in the jet. Strong cooling behind shocks formed in the nonlinear regime can produce emission knots and filaments. In some cases, the modes grow until the jet is disrupted. Distortions in the surface of the jet drive shock spurs into the ambient gas, resulting in longitudinal acceleration. Rapid acceleration and entrainment of ambient gas is also observed if the jet is disrupted.
\end{abstract}

\section{Introduction}

In the last few years, high-resolution images have been obtained for a number of protostellar jets using, for example, the HST. In fact, many of these images have been presented at this meeting (e.g. see Reipurth \& Heathcote, this volume). Perhaps the most exciting aspect of these images is that the internal structure of the jet beam is resolved, in many cases for the first time, allowing the internal dynamics of the jet to be probed. In nearly every case, one finds the optical emission from the interior of the jet is highly complex, associated with knots and filaments of emission, curved 'internal bow shocks', and in some cases kinks and wiggles in the direction of propagation. As an extreme example of how complex the situation can become, I simply refer to the HST image of HH47 which graced the cover of the 
poster book, and which has been described in the literature by Heathcote et al (1996).

It seems clear that in the most complex systems, a variety of physical effects are at work to create the observed patterns of emission. For example, there is little doubt that many, if not all, protostellar jets are time-variable, and undergo periodic eruptions. Less certain is the possibility that the direction in which the jet is launched undergoes large-angle variations, perhaps due to 'precession' of the underlying accretion disk. That such 'pulsing' or changes in direction of the jet beam will lead to structure downstream is obvious: the structure is forced upon the flow by the boundary conditions at the source of the jet. However, it is also well known that the internal dynamics of a supersonic jet beam is rich enough to allow the formation of structure such as knots, filaments, and wiggles simply through dynamical instabilities associated with the jet itself. For example, detailed hydrodynamical studies of the propagation of cooling, overdense jets, beginning with Blondin et al (1990), has shown how Rayleigh-Taylor instabilities associated with dense sheets of gas formed by cooling fragment into dense knots (de Gouviea dal Pino \& Benz 1993; Stone \& Norman 1993a; 1993b; 1994). At the same time, studies of extragalactic jets have shown that various modes of the Kelvin-Helmholtz instability can produce either knots in the jet beam, or large amplitude wiggles leading to disruption at certain frequencies (e.g., see the reviews of Birkinshaw [1991], Hardee [1998]). The latter result seems especially appropriate to the observations of 'precessing' jets, however since extragalactic jets fall in a completely different region of parameter space than do protostellar jets (the former are thought to be both underdense compared to their surroundings and adiabatic, as opposed to overdense and cooling), very little of the previous work can be applied directly to protostellar systems.

Despite the obvious complexity of real systems such as HH47, it seems fruitful to consider the dynamics of an idealized supersonic jet beam in order to assess the importance of dynamical instabilities in forming structures within the beam. It is hoped that with this understanding, more complex situations can be investigated. With this in mind, my collaborators and I have recently finished a detailed analysis of the K-H instability in cooling jets in a paramenter regime appropriate to protostellar systems. In this paper, the major results and implications of this work will be summarized.

\section{Problem Setup}

The simplest model of an idealized jet is shown schematically in Figure 1. The jet is composed of a perfectly collimated, homogeneous stream propagating through a uniform ambient medium. Of course, in this figure the am- 


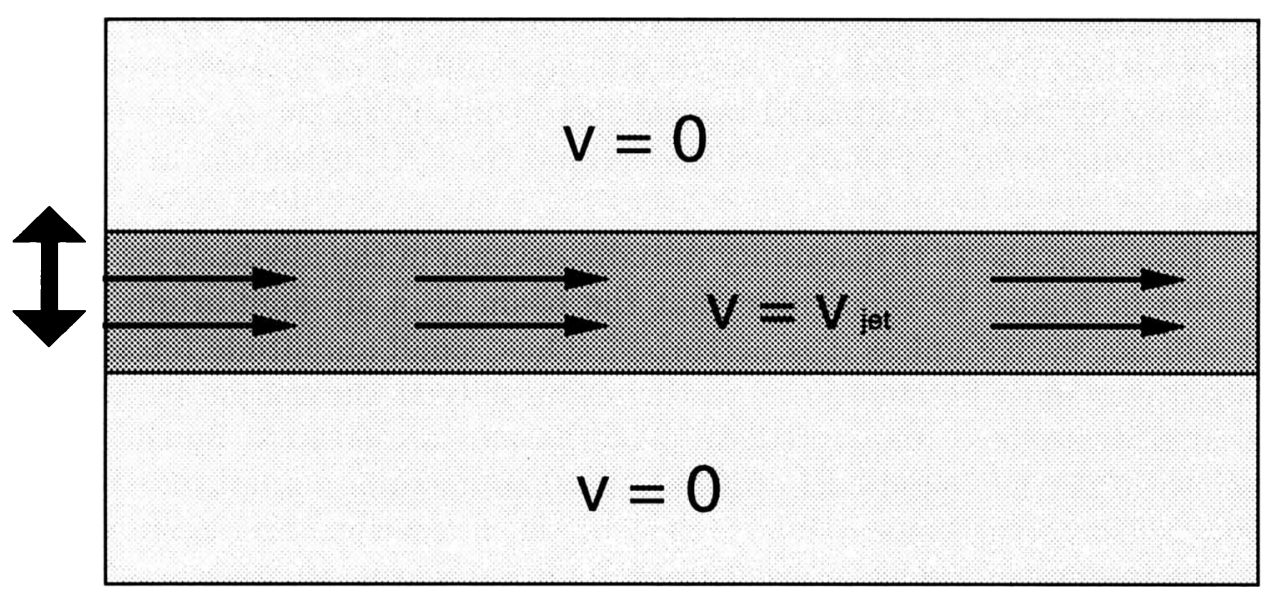

Figure 1. Schematic structure of an idealized protostellar jet beam.

bient medium does not actually represent the background fluid into which the jet propagates, rather the jet beam interacts directly only with a 'cocoon' composed of a mixture of shock processed ambient and jet gas. For this reason, the ambient medium shown in Figure 1 is never entirely uniform or quiescent in a propagating jet, nonetheless this complication is ignored in order to focus on the dynamics of the beam itself.

It is clear there will be K-H instabilities associated with the slip surface which defines the surface of the jet. However, because acoustic waves in the interior of the beam are reflected at the surfaces, the jet beam forms a resonant cavity which amplifies certain frequencies better than others. The result is that certain modes of the $\mathrm{K}-\mathrm{H}$ instability will grow faster than others, and ultimately will dominate the jet. Our goal is to calculate which modes grow fastest in a protostellar jet, at what rate, and to understand what happens when the modes become nonlinear.

As mentioned in the Introduction, the analysis of the K-H instability in adiabatic jets is well developed. In cooling jets, Bührke et al (1988) were the first to suggest that axisymmetric pinch modes of the instability might be responsible for producing the chain of knots observed down the jet beam observed in such systems as HH34. However, the knot spacing and proper motions do not agree well with the predictions of the linear theory. Still, only recently has the evolution of the symmetric modes in cooling and overdense jets been thoroughly studied (see Massaglia et al, this volume). Moreover, even if the pinch modes are found not to be the primary source of symmetric knots, the asymmetric modes may still be important to produce wiggles and kinks in the beam. Finally, K-H instabilities are ultimately 
responsible for the production of "turbulent mixing layers" which lately have become the fashion to explain the acceleration of molecular bipolar outflows by highly collimated optical jets. Thus, if we are to understand such mixing layers we must first understand the $\mathrm{K}-\mathrm{H}$ instability in cooling jets.

How does the K-H instability initially develop in the idealized jet beam shown in Figure 1? First, note that the beam is in an equilibrium state: it will not evolve unless it is perturbed. Thus, small amplitude linear perturbations must be introduced in the jet, usually at the nozzle, by adding a small transverse velocity (these perturbations are denoted by the thick arrow on the left hand side of Figure 1). The maximum amplitude of the transverse perturbation velocity must be much less than the sound speed in the interior of the jet in order to excite linear waves. If larger amplitude perturbations are used, they will already be of nonlinear amplitude, and will immediately produce shocks and distortions of the beam which have no relation to the resonant frequencies characteristic of the beam itself, but simply reflect the nature of the imposed variations. Fundamentally, the purpose of the perturbations is to introduce acoustic noise into the jet which can seed the instability.

Of course, our goal is to understand how the jet responds to the perturbations. If the jet is very overdense compared to its surroundings, i.e. if $\rho_{\text {jet }} / \rho_{\text {ambient }} \geq 100$, where $\rho_{\text {jet }}$ and $\rho_{\text {ambient }}$ are the mass densities of the jet beam and ambient gas (remember this means cocoon) respectively, then the jet is effectively ballistic, and it will propagate without much notice of the ambient medium. In this case, it is clear that the maximum transverse displacement of the jet beam grows linearly with distance down the jet for a constant amplitude (transverse) perturbation. On the other hand, if $\rho_{\text {jet }} / \rho_{\text {ambient }}<100$, the response of the jet beam to the perturbation is much more complex, and can only be computed by considering the linearized equations of hydrodynamics which describes the evolution of the perturbations. As we shall see in the next section, this leads to the maximum transverse displacement of the jet beam growing exponentially with distance down the jet. In principle, with high enough resolution, the distinction between linear and exponential growth of the amplitude of 'wiggles' in observations of the beam would indicate whether simple precession or hydrodynamical instability were responsible.

\section{Linear Analysis}

The evolution of small amplitude perturbations in a supersonic jet is governed by the linearized equations of hydrodynamics. If the initial equilibrium state is uniform (as it is in our case), a Fourier analysis can be used 
to calculate the dispersion relation for waves in the system, i.e. $k=k(\omega)$, where $\omega$ and $k$ are the frequency and wavenumber of disturbances. Modes with complex wavenumbers then represent either exponentially growing or decaying waves, with the former resulting in instability. Generally, finding analytic solutions to the dispersion relation in closed form at arbitrary frequencies is not possible, therefore numerical root finding techniques are required to trace the solution over a broad range of perturbation frequencies.

Interested readers are referred to Hardee \& Stone (1997, hereafter Paper I) for a complete discussion of the details of the analysis. The only important point to stress here is that some choice must be made for the net cooling function used in the analysis, and that the net cooling must be zero in the initial equilibrium state. In Paper I, two different cooling functions were adopted: (1) the cooling rate appropriate to photoionized gas described by MacDonald \& Bailey (1981, hereafter MB), and (2) the cooling rate for interstellar gas given by Dalgarno \& McCray (1972, hereafter DM). The former is appropriate for the study of Seyfert jets, the latter for the systems of interest here, namely protostellar jets. In simulations of propagating jets, Stone \& Norman (1993a) found significant differences in the nonlinear evolution of the system if a more accurate non-equilibrium cooling function were adopted, however the linear analysis of $\mathrm{K}-\mathrm{H}$ modes with more complex cooling functions is simply intractable. Since we are only interested in linear amplitude perturbation away from the equilibrium state, linearized cooling functions which approximate either the MB or DM cooling curves in the neighborhood of the equilibrium temperature are all that are required.

The results of the linear analysis are described in Paper I. Figure 2 shows the results for a Mach 20 jet which is 10 times denser than its surroundings (here and throughout this paper, the Mach number is defined as the ratio of the jet velocity to the sound speed in the ambient medium). Plotted are the dimensionless growth rate for the both the fundamental surface wave, and higher order body modes, as a function of dimensionless frequency (see paper I for a description of the classification of $\mathrm{K}-\mathrm{H}$ modes into surface and body waves). The top panel plots these quantities for the adiabatic jet, the middle for a jet with DM cooling, and the bottom for a jet with $\mathrm{MB}$ cooling. Comparison between the panels immediately demonstrates the differences radiative cooling makes to the evolution of $\mathrm{K}$ $\mathrm{H}$ modes in supersonic jets. For example, in the adiabatic and MB cooling jet, the growth rate of the surface wave has a maximum at some frequency (called the resonant frequency). We might expect that this resonant frequency, when it exists, will dominate the nonlinear evolution. The dots plotted on each panel are the growth rates measured from the initial linear 

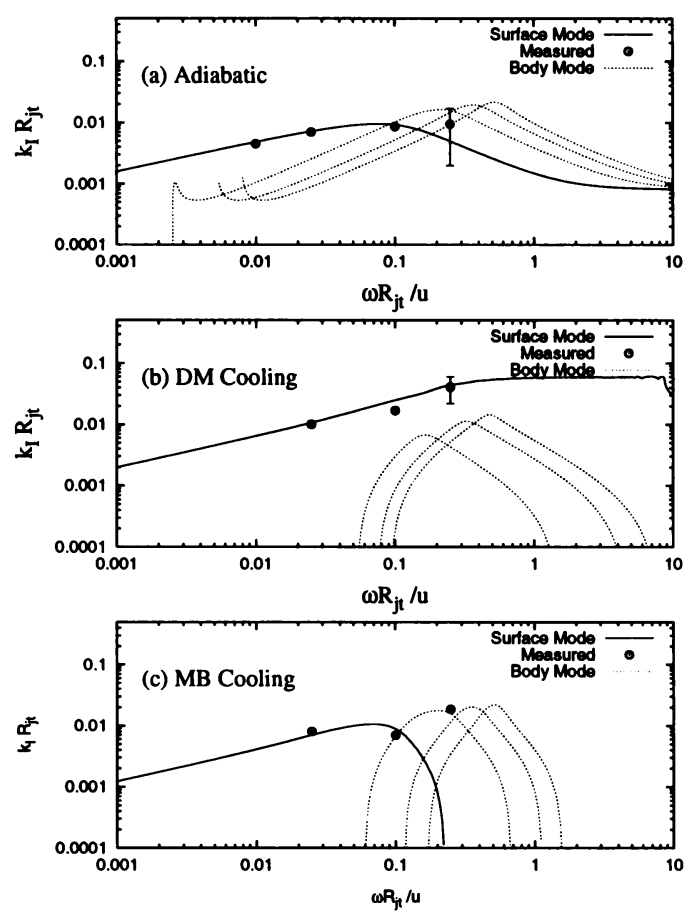

Figure 2. Growth rate of some asymmetric K-H modes in a Mach 20 protostellar jet.

evolution in a nonlinear hydrodynamical simulation of the instability - they provide both a code test and a check on the linear analysis. Generally, very good agreement is found between the two.

\section{Nonlinear Hydrodynamical Simulations}

In order to study the growth and saturation of K-H modes in a cooling jet in the nonlinear regime, numerical methods which solve the equations of hydrodynamics, combined with the full nonlinear cooling function over a wide range of temperatures, are required. We have used a two-dimensional (planar symmetric) hydrodynamics code based on the PPM algorithm to study the evolution of asymmetric modes in slab jets. Typically, the computational volume of size $20 R_{j t}$ in the transverse direction, and $400 R_{j t}$ in the longitudinal direction (with $R_{j t}$ the jet radius) is divided into up to $200 \times 800$ zones. Nonuniform zones are used in the transverse direction, so that typically the numerical resolution within the jet beam is 20 zones per jet radius. The equilibrium jet is initialized across the entire mesh (with 


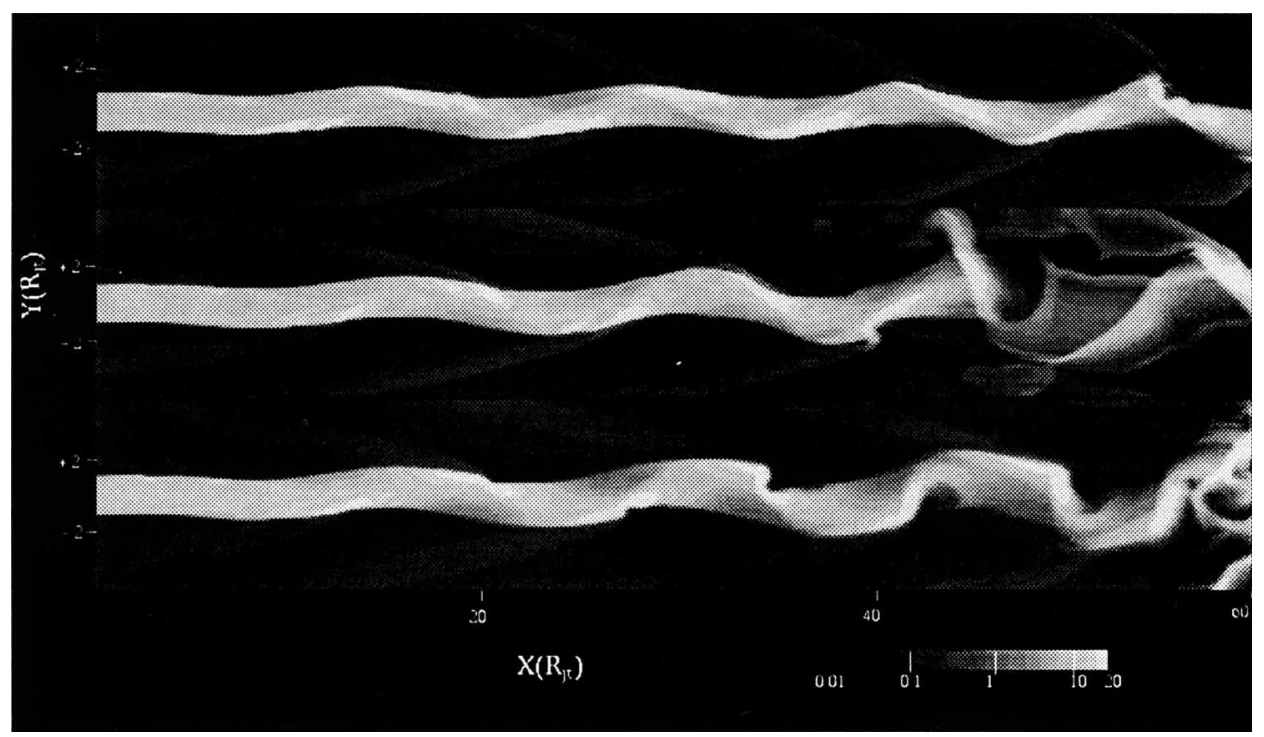

Figure 3. $\log$ (density) for a Mach 5 adiabatic (top), DM cooling (middle) and MB cooling (bottom) jet perturbed at a frequency of $\omega R_{j t} / u=0.4$. Note the formation of dense knots and filaments in the cooling jets.

inflow boundary condition on the left hand side of the grid, and outflow on the right), the jet is perturbed at the nozzle, and the evolution is followed for several hundred dynamical times (sound crossing times of the jet beam). The results of a series of simulations using different perturbation frequencies and cooling curves is given in Stone, Xu, \& Hardee (1997, hereafter Paper II). We summarize some of the basic results here. Axisymmetric (two-dimensional) simulations of the pinch modes is described by Massaglia et al (this volume), and fully three-dimensional simulations of adiabatic jets are presented by Hardee et al (1997).

Figure 3 plots grayscale images of the logarithm of the density at late time for a Mach 5 jet computed with an adiabatic equation of state, and $\mathrm{DM}$ and $\mathrm{MB}$ cooling driven at a frequency $\omega R_{j t} / u=0.4$ (near to the maximum growth rate of the surface wave, here $u$ is the axial velocity of the jet) using a numerical resolution of 40 zones across a jet diameter. In each case, internal structure associated with sinusoidal oscillation grows strongly, forming a sequence of opposing shocks in the jet. Note that the DM cooling jet appears to disrupt at a lesser distance, $\approx 45 R_{j t}$, than the adiabatic and MB cooling jets. In the cooling jets, the postshock gas becomes very dense. The corrugations in the edge of the jet produce oblique shock spurs in the ambient gas oriented in a sequence of opposing steps. Interestingly, the knots themselves are subject to Rayleigh-Taylor instabilities, and slowly 


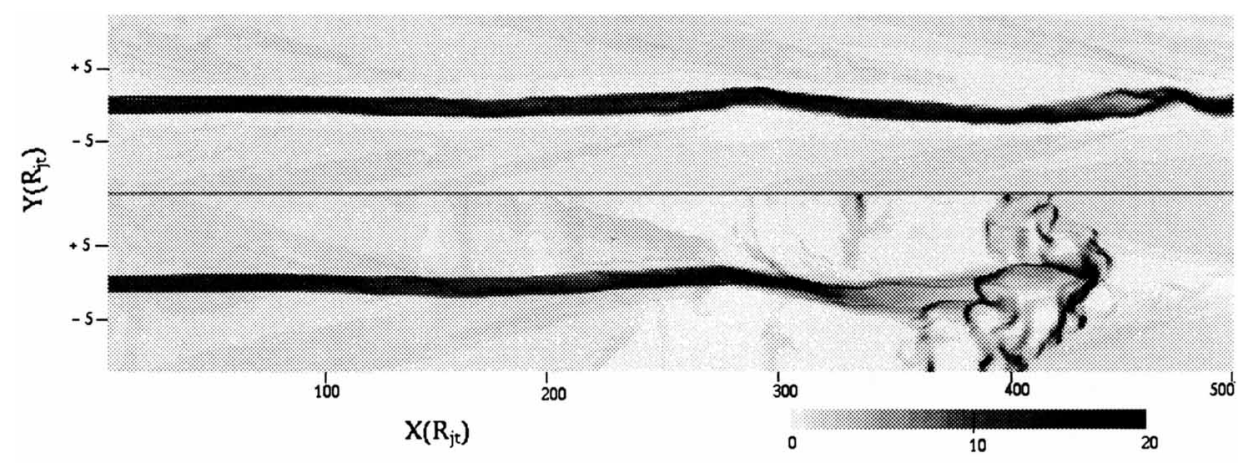

Figure 4. $\log$ (density) for a Mach 20 adiabatic (top) and DM cooling (bottom) jet perturbed at a frequency of $\omega R_{j t} / u=0.025$.

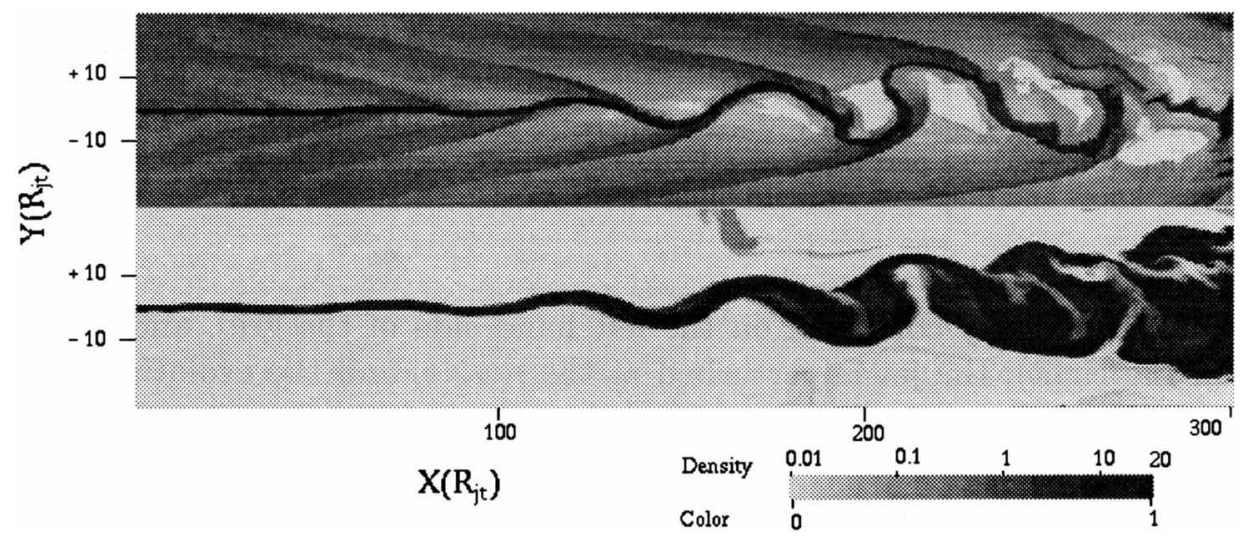

Figure 5. Log(density) (top) and 'color' (Lagrangian tracer) variable (bottom) for a Mach $5 \mathrm{DM}$ cooling jet perturbed at a frequency of $\omega R_{j t} / u=0.1$. Strong mixing is evident once the jet disrupts.

break up at late times. For comparison, in Figure 4 we plot grayscale images of the density in Mach 20 adiabatic and DM cooling jets driven at a low frequency of $\omega R_{j t} / u=0.025$. Unlike jets perturbed at high frequency, jets perturbed at this low frequency have a long linear evolution phase. The $\mathrm{DM}$ cooling jet is predicted by the linear analysis to have a growth rate $\approx$ $30 \%$ larger than the adiabatic jet, and, in fact, the adiabatic jet remains relatively well collimated whereas the DM cooling jet is disrupted and forms high density knots associated with working surfaces in the ambient gas. The dense knots result from strong cooling effects.

In Figure 5 we plot grayscale images of the logarithm of the density and 
the color variable for simulations of a Mach 5 jet computed with the DM cooling curve. The jet is perturbed at a relatively low frequency of $\omega R_{j t} / u=$ 0.1 , and the result is shown after 200 dynamical times when it has reached a quasi-stationary state. This jet shows a long linear evolution phase and an overall sinusoidal pattern associated with the growth of surface waves. The surface wave grows to large amplitude at large distances from the nozzle, and disrupts the jet. Shock spurs driven into the ambient gas by the sinusoidal jet oscillations are evident. In addition to the surface wave there are oblique structures within the jet. These structures are manifested primarily as dense knots or filaments oriented on either side of the jet in an alternating pattern.

By using a Lagrangian tracer variable which can distinguish between the jet and ambient gas, we have measured the rate of mixing between these two fluids for the jet shown in Figure 5 . We find that little mixing occurs near the jet nozzle, i.e., inside $x=100 R_{j t}$ where the waves are still in the linear regime. Mixing of the ambient gas starts where the perturbation reaches significant amplitude and then grows exponentially with the amplitude of the perturbation. This spatial growth of the mixing fraction appears to be in agreement with the results of Bodo et al. (1995). By tracking the momentum of the ambient gas, we also find that acceleration of the ambient gas occurs within $50 R_{j t}$ of the nozzle, even before the jet disrupts and mixes significantly. Figure 5 shows that in this region strong shock spurs are driven into the ambient gas by the sinusoidal distortion of the jet. These shock spurs result in acceleration of the ambient gas. Although most previous discussions of the entrainment and acceleration of ambient gas along a jet beam have focused on turbulence either in a mixing layer, or in the turbulent "wake" of internal bow shocks in pulsed jets, our simulations show that shock trains in the ambient gas can result in acceleration of ambient gas. These shock trains may be produced by the K-H instability, as our simulations demonstrate, or they may be the wings of internal bow shocks produced by pulsing the jet.

\section{Summary}

Asymmetric modes of the $\mathrm{K}-\mathrm{H}$ instability may be an important factor, along with other effects such as time-variability or 'precession', in producing complex structure within protostellar jets. To address this issue, detailed investigation of the properties of the instability in cooling, overdense jets, as described here, are required.

In Paper I, we presented solutions to the dispersion relation in the linear regime for $\mathrm{K}-\mathrm{H}$ modes in a cooling jet over a wide range of perturbation frequencies. Our results indicated that the linear growth rates of waves 
in a cooling jet are substantially different from those of an adiabatic jet. Depending on the details of the cooling function adopted, the growth rate of waves could be either enhanced or reduced relative to those on the adiabatic jet.

In Paper II, we have continued this analysis by using time-dependent hydrodynamical simulations to follow the growth of unstable waves into the nonlinear regime. Growth accompanying low frequency and long wavelength perturbations can dominate the structure of the jet at late times producing large amplitude sinusoidal oscillations of the jet beam. Once the amplitude of the oscillations exceeds several jet radii, the jet is disrupted. On the other hand, higher frequency perturbations can excite body waves which result in the formation of internal sinusoidal structures. Strong internal shocks produced by these body waves can form a pattern of dense knots which are staggered asymmetrically along the length of the jet. We find that internal shocks produced by relatively short wavelength body waves can coexist on a jet with a much longer wavelength slowly growing sinusoidal oscillation of the entire jet. We also find that small amplitude oscillations of the jet associated with a growing sinusoidal surface or with a strong body wave can drive shock spurs into the ambient gas, leading to an alternating pattern of knots and shock spurs. These shock spurs can accelerate the ambient gas without mixing it with the jet fluid. Disruption of the jet by the $\mathrm{K}-\mathrm{H}$ instability produces strong mixing and entrainment of ambient gas, and the formation of dense knots of shocked ambient gas swept up by jet material.

Structures observed in our simulations such as low amplitude wiggles in the jet, dense knots formed asymmetrically with respect to the center of the jet, and an alternating pattern of shock spurs driven into the ambient gas are all reminiscent of high resolution observations of protostellar jets. On the other hand, for a typical protostellar jet, dynamical periods associated with the inner $(r<1 \mathrm{AU})$ regions of an accretion disk are much less than the period of perturbations to which the entire jet can respond dynamically (which is about 200 years). We therefore expect protostellar jets to be dominated by the growth of body waves and internal asymmetric structures rather than the surface waves.

Of course, real protostellar jet systems will be three-dimensional. The asymmetric modes studied here are the analogue of the helical modes on a $3 \mathrm{D}$ jet. However, additional modes are also possible in 3D. In a companion work Hardee et al (1997) have presented a study of the K-H modes in a fully 3D supersonic jet including a dynamically important axial magnetic field. Actually, most models for driving jets from accreting protostars predict that the magnetic field far downstream in the outflow will be primarily toroidal the stability properties of a cooling, toroidally magnetized jet have yet to be analyzed, although see Todo et al (1993) for a simulation of an adiabatic 
jet. Finally, it also important to remember that theoretical studies such as those described here are necessarily limited to idealized jets like that shown in Figure 1. If the jet instead has significant radial structure, e.g. it is composed of a shear layer of finite thickness, the details of the results (e.g. precise values of growth rates, etc.) will be changed, although probably the general character of unstable modes will not. It is hoped that detailed theoretical investigations of jet dynamics will ultimately enable detailed modeling of specific observations.

Acknowledgements: I thank my collaborators Phil Hardee and Jianjun $\mathrm{Xu}$ for their many contributions to this work, and I gratefully acknowledge support from the NASA Astrophysics Theory Program under grant NAG-52886.

\section{References}

Birkinshaw, M. 1991, in Beams and Jets in Astrophysics, ed. P. Hughes, (Cambridge: CUP), 278

Blondin, J.M., Fryxell, B.A., \& Königl, A. 1990, ApJ 360, 370

Bodo, G., Massaglia, S., Rossi, P., Rosner, R., Malagoli, A., \& Ferrari, A., 1995, A\& A 303,281

Bührke, T., Mundt, R., \& Ray, T.P. 1988, A\&A 200, 99 (BMR)

Dalgarno, A., \& McCray, R.A. 1972, ARA\&A 10, 375 (DM)

de Gouviea dal Pino, E.M., \& Benz, W. 1993, ApJ 410, 686

Hardee, P.E., \& Stone, J.M. 1997, ApJ 483 (Paper I)

Hardee, P.E., Stone, J., Rosen, A.: 1997, in Low Mass Star Formation - from Infall to Outflow, poster proceedings of IAU Symp. No. 182, eds. F. Malbet \& A. Castets, Observ. de Grenoble, 1997, p. 132

Hardee, P.E. 1998, in The 12th Kingston Meeting on Theoretical Astrophysics: Computational Astrophysics, eds. D. Clarke \& M. West (San Francisco: ASP) in press.

Heathcote, S., Morse, J., Hartigan, P., Reipurth, B., Schwartz, R.D., Bally, J., \& Stone, J.M. 1996, AJ 112, 1141

MacDonald, J., \& Bailey, M.E. 1981, MNRAS 197, 995 (MB)

Massaglia, S., Trussoni, E., Bodo, G., Rossi, P., \& Ferrari, A. 1992, A\&A 260, 243

Stone, J.M., \& Norman M.L. 1993a, ApJ 413, 198

Stone, J.M., \& Norman M.L. 1993b, ApJ 413, 210

Stone, J.M., \& Norman M.L. 1994, ApJ 420, 237

Stone, J.M., Xu, J., \& Hardee, P. 1997, ApJ 483 (Paper II)

Todo, Y., Uchida, Y., Sato, T., \& Rosner, R. 1993, ApJ 403, 164 